\title{
THE APPLICATION OF (RCA 1P 28)-PHOTOMULTIPLIER TUBES TO THE DETECTION OF WEAK LIGHT INTENSITIES
}

\author{
by F. BOESCHOTEN, J. M. W. MILATZ and C. SMIT \\ Physisch laboratorium der Rijksuniversitcit, Utrecht, Nederland
}

\section{Synopsis}

Two methods are discussed of measuring weak light intensities (varying but slowly with time) with the aid of a photomultiplier tube.

$1^{\circ}$. the light-chopping method in combination with a phase- and frequency-sensitive measuring device (i.c. alternating current galvanometer 1) 2) 3) 4) ${ }^{16}$ ).

$2^{\circ}$. the counting method, as used, for instance, in scintillation counters ${ }^{4}$ ) ${ }^{5}$ ).

Especially the factors determining the detection limit and the reproducibility of these methods are investigated, notably: the internal amplification factor, the quantum efficiency and the dark current of the photomultiplier. These factors were found to be different between ten RCA 1 P28 tubes operating under the same condition, with an average spread of about two times and an extreme spread of ten times.

The output-current generated by a lightflux of constant intensity, proved to be constant in time within $1 \%$ (the error of measurement) when a well stabilized high voltage source is used. The dark current however, turned out to vary very irregularly with time.

The noise of the photo- and dark-current measured within a narrow band about $50-\mathrm{Hz}$ appeared to exceed the calculated value by a factor $2-3$, which may be due to a Flicker effect.

The optimum operation conditions for measuring weak intensities were found to be obtainable with an uniform voltage drop amounting to $60 \mathrm{~V}-70 \mathrm{~V}$ over each stage.

The effect of decreasing the thermionic current (the most important component of the dark-current) by cooling the tube with liquid air, on the useful sensitivity was investigated and so was the alteration of the spectral response curve as a function of the temperature.

$\S 1$. The methods and expedients of measuring. The properties of multiplier tubes were investigated by applying the following methods of measuring:

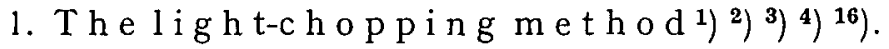

The lightflux is interrupted with a frequency of $50 \mathrm{~Hz}$ before it reaches the photocathode of the multiplier, which is connected to an amplifier with two stages (input resistance $10 \mathrm{M} \Omega$, with Johnson noise at $T=300^{\circ} \mathrm{K} \gg$ shot noise in grid-and plate current). The amplified alternating current is led to an a.c. galvanometer.

When recording deflections without special precautions an accuracy better 
than $1 \%$ is obtained. Little inconvenience is caused by the dark-current and by non- $50 \mathrm{~Hz}$ interrupted light.

The deflection error of the galvanometer given in terms of an effective noise tension on the first grid of the amplifier is theoretically given by ${ }^{9}{ }^{4}$ ):

$$
\widetilde{\Delta V}=\sqrt{\left(4 k T R+(2 n /(n-1)) G e I R^{2}\right) \pi / 4 \tau_{0}} .
$$

$k=$ Boltzmann constant; $T=$ temperature; $e=$ charge of an electron.

The first term between the brackets represents the Johnson noise in the input resistance $R$, at temperature $T$.

The second term is the shot noise in the outputcurrent $I$ of the multiplier; the factor $n /(n-1)$ is due to statistical fluctuations in the secondary enission factor $n . G$ is the internal amplification factor.

With the used input resistance of $10 \mathrm{M} \Omega$ parasitic capacities play no part in the impedance of the grid circuit at $50 \mathrm{~Hz}$. Shot noise in grid- and plate current of the first tube play no part either. The factor $\pi / 4 \tau_{0}$, with $\tau_{0}=$ the time of indication of the galvanometer, represents the "spectral bandwidth" $\Delta f$ of the galvanometer response; for a.c. signals $\Delta f \approx 1 \mathrm{~Hz}$ in our case. Filling in the values of the constants expressed in Giorgi units yields:



Lest the Johnson noise exceeds the shot noise of the multiplier current, it is necessary that $20(n /(n-1)) G I R \gg 1$. In the next section it will be shown that this condition is easily fulfilled.

- This set can also be used for measuring dark-current and non-interrupted light. Then a double poled $50-\mathrm{Hz}$ interruptor (Carpenter relais type $3 \mathrm{Gl}$ ) is to be placed in the grid circuit of the pre-amplifier.

2. The counting method.

The electronic part of the apparatus resembles that used in scintillation counters. The multiplier is connected to a broad band amplifier, discriminator, scalers and a mechanical register. An oscilloscope is connected to the amplifier output too. The light flux is now measured by counting the outputpulses initiated by the primary photo-electrons, passing the discriminator during a well determined interval of time.

The discriminator can be replaced by a differential discriminator in order to determine directly the pulse height distribution.

In both cases we used a high voltage supply, developed in our laboratory for multipliers, which is continuously adjustable from $500 \mathrm{~V}-2000 \mathrm{~V}$; maximum current $5 \mathrm{mAmps}$. The high voltage is constant within $0.1 \%$.

The following lightsources were used:

1. A neon discharge tube with constant emission within $1 \%$, fed by $210 \mathrm{~V}$, $50-\mathrm{Hz}$, in combination with $4 \mathrm{~mm}$ VG 2 Schott filter. The discharge column is screened in such a way that a 50 (not 100) $\mathrm{Hz}$ modulated light flux results. 
Mainly light in the spectral region of $5000 \AA-5500 \AA$ is emitted to the total amount of $4( \pm 1) \cdot 10^{-9}$ watt in a nearly parallel beam of $3.5 \mathrm{~mm}^{2}$ diameter. A disadvantage in using this lightsource is that the spectral sensitivity curve of the multiplier descends steeply at $5000 \AA$.

2. An incandescent lamp of 0.5 watts, fed by battery. When an UG 1 Schott filter was used mainly blue light was obtained in the region of $4000 \AA-3500 \AA$ where the multiplier has nearly its maximum spectral sensitivity. The constancy was better than $0.5 \%$. A nearly parallel and homogeneous beam of light was obtained which overflowed the area of the photocathode, so that the position of the multiplier was not very critical.

\$2. Survey of properties and factors to be measured. Of the quantities, determinative for the operating of a multiplier, which as a matter of fact determine the utmost sensitivity achieved by a multiplier, we may make up the following survey ${ }^{8}{ }^{6}{ }^{6}{ }^{4}$ ):

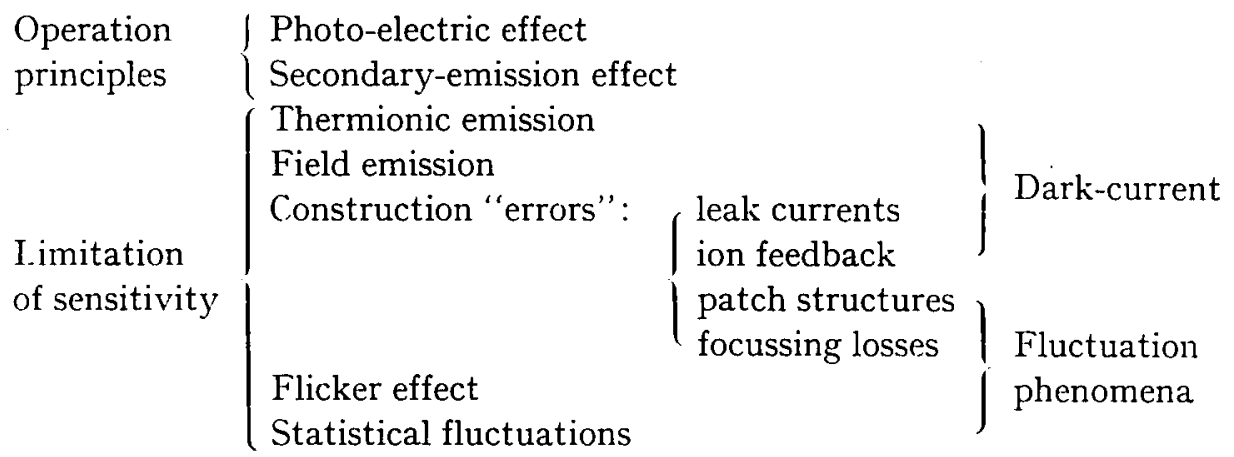

Since fluctuation phenomena in the dark-current limit the least detectable amount of light, it was important to know how to minimize these quantities in order to obtain the multiplier operating with optimum sensitivity. In the next section our results will be given with regard to the following quantities:

I. Constancy of the output current.

II. Sensitivity of the photocathode as a function of the manner of illumination.

III. Internal amplification factor.

IV. Linearity.

V. Quantum efficiency.

VI. Dark-current.

VII. Noise in dark-current and photo-current.

VIII. Optimum voltage drop over the stages.

XI. Effects of cooling with liquid air.

When the experimental values mentioned in the following section are considered it should be born in mind that they are averaged over ten multipliers, with a spread of about a factor two, and in extreme cases a factor ten. 


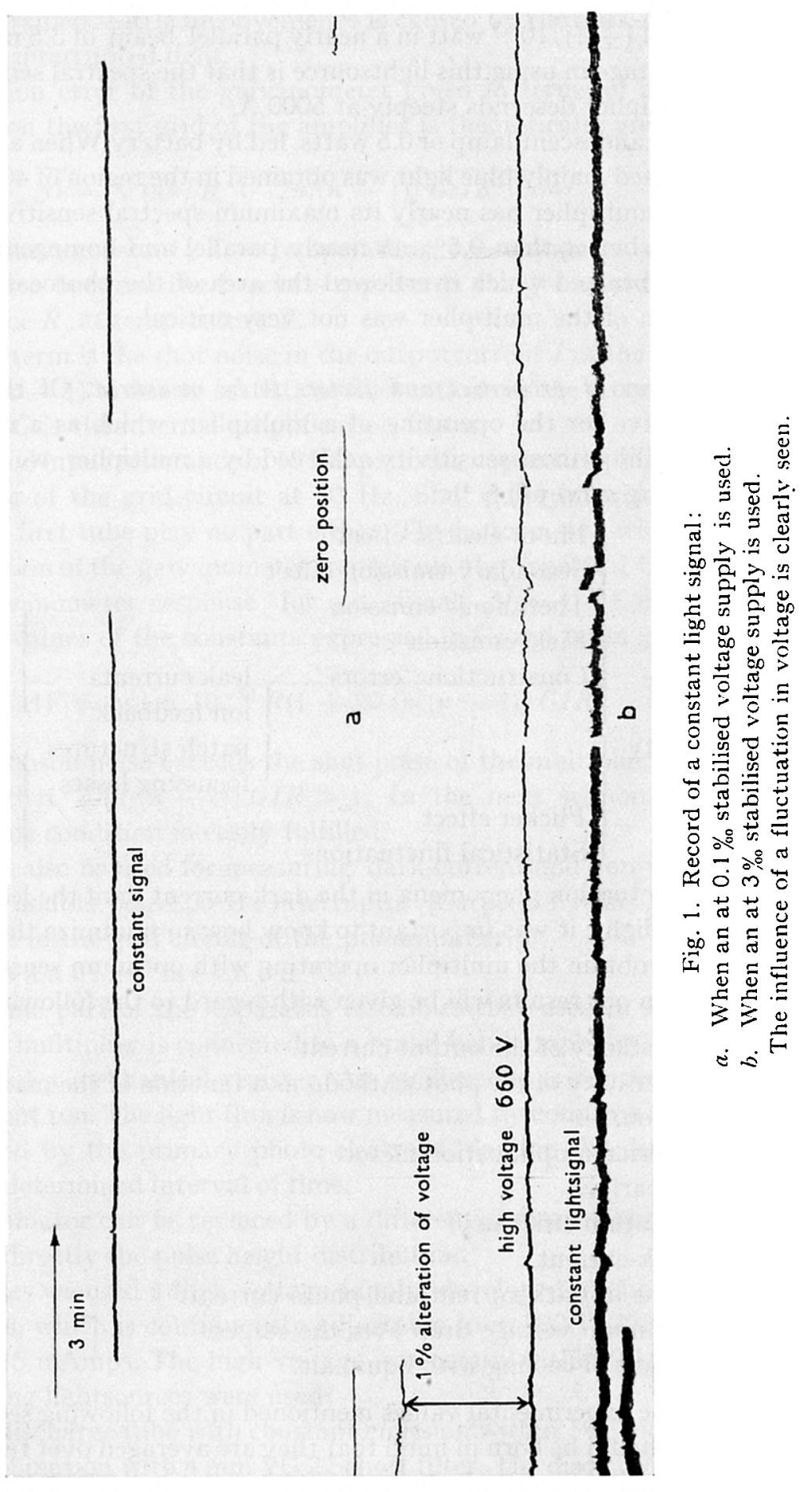


A tube that differs considerably from the others in one respect needs not do so with regard to other quantities. For instance one tube had an amplification factor which was 7 times lower than that of an average tube, but still had the same signal-to-noise ratio.

\$3. Experimental results. I. C on stancy of the out put phot o-c u r r e n t ${ }^{4}$ ). The output photo-current, averaged over the measuring time, proved to be constant within $1 \%$ (accuracy of the measurements), if

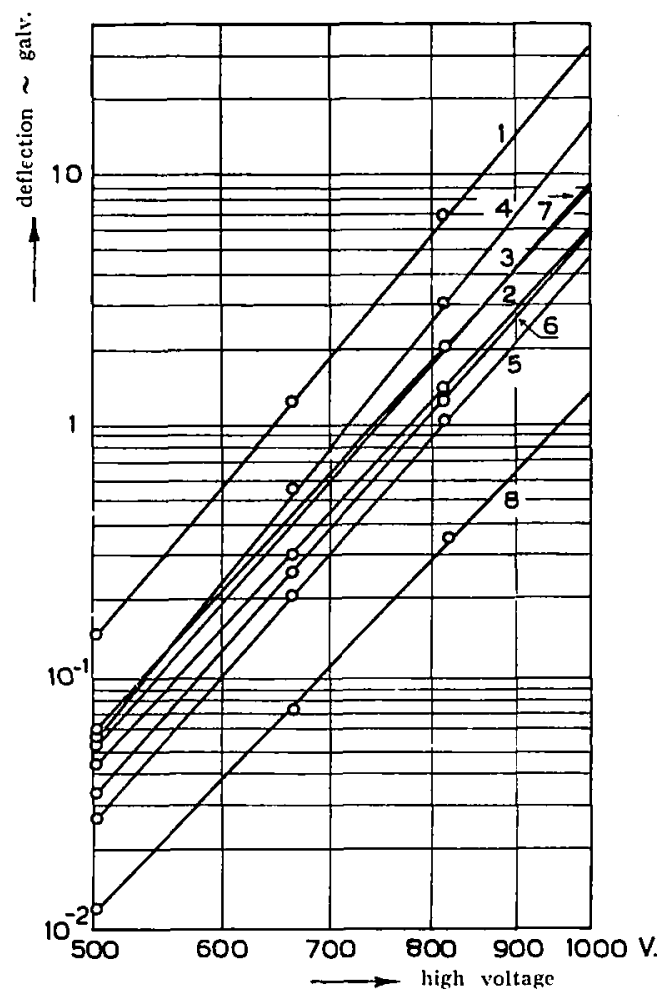

Fig. 2. The a.c. galvanometer deflection as a function of the used voltage for a number of photomultipliers.

the multiplier was illuminated by a lightsource of sufficient constancy. This result was obtained with both methods of measuring at short period (few seconds) as well as at long period (few hours).

In order to obtain this result, it is necessary that:

1. The voltage drop over the stages remains constant within $0.2 \%$.

2. The generated output current is lower than 10 micro-amperes; otherwise fatigue phenomena may arise.

3. The voltage drop per stage is less than $100 \mathrm{~V}$.

Fig. 1 shows the recorded deflection of the a.c. galvanometer for a constant 
light flux, with a "good" stabilized high voltage supply and with a "bad" one respectively, that had still a constancy of $3 \%$.

II. Manner of illuminating the photocathod $\left.\left.\mathrm{e}^{10}\right)^{6}\right)^{13}$ ). In order to get a reproducebility better than $1 \%$ it is necessary to illuminate the photocathode homogeneously. As the local sensitivity is not constant over the surface of the cathode the position of the light spot will be very critical and a slight shift will change the photo-current considerably. When a circular lightspot of $1 \mathrm{~mm}^{2}$ area was moved over the cathode surface, the maximum and minimum local sensitivity seemed to differ a factor 5 . The expected dependence of this variance on the dimensions of the light-spot may explain why different results were mentioned in the litterature.

So it is of importance to fill the cathode area homogenously with light; moreover this has the advantage that a given amount of light causes relatively less fatigue effects.

III. Internal a mplification $f$ a ctor $\left.{ }^{6}\right)^{7}$ ). The dependence of the multiplier output current, that is of the amplification factor, on the total voltage $\mathrm{V}$ over the multiplier seems to obey $I=C V^{a}, C$ is a constant, for normal operating conditions, $a$ is a constant for every tube $(7<a<8)$ (see fig. 2).

The internal amplification factor was determined by using the multiplier, illuminated by a $50-\mathrm{Hz}$ modulated lightsource, as a phototube and measuring the current arriving at the first dynode by the a.c. method. After that the output current of the tube, now again used as multiplier, was measured with the sensitivity control of the amplifier in the most insensitive position and with known attenuation of the illumination. The internal amplification found in this way, varied maximally a factor 6 between the investigated tubes (fig. 2).

The average gain found in this way corresponds with the values stated by the manufacturer.

It was found, however, that the amplification factor varies (mostly decreases) very slowly in the course of time, especially when the multiplier was frequently used. This variance may in extreme cases amount to a factor 2 in one year.

If the multiplier - as in our case - is combined with an amplifier, the internal amplification factor is of no practical importance.

IV. Lin e arit y. The deflection of the galvanometer with the light chopping method, as well as the counting rate with the counting method turned out to be within $2 \%$ (accuracy of the measurement) proportional with the incident lightflux $\gtrsim 10^{-13}$ Watt (for these signals noise in the photo- and thermionic currents was already visible).

V. $Q$ u a n t u m-e f $f$ i c i e $\left.n c y^{6}\right)^{7}$ ). We define the quantum-efficiency as the mean number of photoelectrons released by one incident photon averaged over the area of the photocathode, with homogenous illuminiation. 
The quantum-efficiency was determined by operating the multiplier as a phototube and illuminating with a signal of known intensity and spectral composition. For wavelengths of $5000 \AA-5500 \AA$ a quantum efficiency was found of about $2 \cdot 10^{-3}$. In the region of maximum energy sensitivity the quantum-efficiency amounts to 0.01-0.02.

VI. Dark-curren $\left.t^{6}\right)^{8}$ ). The dark-current was measured directly with a sensitive d.c. galvanometer and also with the above mentioned methods. With the a.c. method it was possible to measure the dark current in an indirect way by recording its $50-\mathrm{Hz}$ fluctuations, these being supposed to be caused by the shot-effect ${ }^{*}$ ).

It is generally supposed that the dark-current is principally composed of three components:

1. Ohmic leakage, preponderant at $40 \mathrm{~V}-80 \mathrm{~V}$ per stage, in some cases still of importance at $100 \mathrm{~V}$ per stage.

2. Amplified thermionic emission, preponderant at $60 \mathrm{~V}-100 \mathrm{~V}$ per stage.

3. Regenerative ionization appearing at $80 \mathrm{~V}$ per stage and causing instability at voltages greater than $110 \mathrm{~V}$ per stage.

The influence of the total voltage over the multiplier on the dark-current will be treated in section VJII. The dark-current is not constant in the course of time; on the contrary there is a great variation with time. Directly after applying the voltage to the multiplier this variation often may amount to a factor 5 in the first hour; after using the multiplier for hours it is still for instance $30 \%$ per hour. This variation depends on the pretreatment of the tube. After the multiplier is illuminated with daylight but without voltage,

TABLE I

\begin{tabular}{|l|c|c|c|}
\hline \multicolumn{1}{|c|}{ detecting instrument } & $\begin{array}{c}\text { voltage } \\
\text { per stage }\end{array}$ & $\begin{array}{c}\text { output-dark } \\
\text { current }\end{array}$ & $\begin{array}{c}\text { number of prinary } \\
\text { dark-current electrons }\end{array}$ \\
\hline d.c. galvanometer & $\begin{array}{r}100 \mathrm{~V} \\
80 \mathrm{~V}\end{array}$ & $\begin{array}{c}1.5 \cdot 10^{-3} \mathrm{~A} \\
3 \cdot 10^{-9} \mathrm{~A}\end{array}$ & {$\left[(1.5 \pm 0.5) 10^{4}\right]$} \\
& & & {$\left[(1.2 \pm 0.5) \cdot 10^{4}\right]$} \\
a.c. galvanometer "indirect" & $120 \mathrm{~V}$ & & $(3 \pm 1.5) \cdot 10^{3}$ \\
& $100 \mathrm{~V}$ & & $(2 \pm 1) \cdot 10^{3}$ \\
& $85 \mathrm{~V}$ & & $(1.5 \pm 0.75) \cdot 10^{3}$ \\
& $70 \mathrm{~V}$ & & $(1 \pm 0.5) \cdot 10^{3}$ \\
interruptor + a.c. galv. & $85 \mathrm{~V}$ & $6 \cdot 10^{-9} \mathrm{~A}$ & {$\left[(1.8 \pm 0.5) \cdot 10^{4}\right]$} \\
& $70 \mathrm{~V}$ & $3 \cdot 10^{-9} \mathrm{~A}$ & {$\left[(3 \pm 0.5) \cdot 10^{4}\right]$} \\
counting device & $70 \mathrm{~V}$ & & $(5 \pm 3) \cdot 10^{3}$ \\
\hline
\end{tabular}

*) First the dark-current noise is recorded, which is $\sim \sqrt{ } N_{0}$ ( $N_{0}=$ number of primary darkcurrent electrons/sec); after that the multiplier is illuminated with a very small amount of chopped light, this causes a deflection of the galvanometer $\sim N_{1}\left(N_{1}\right.$ number primary photoelectrons/sec.) as well as an increased noise $\sim \sqrt{ }\left(N_{1}+N_{0}\right)$. Because it is known how many electrons correspond with a deflection, $N_{1}$ and $N_{0}$ may be calculated. This method is treated in more detail in the next section.

Physica XX 
it is increased considerably; after cooling it remains some days smaller than normal, although the multiplier has already been warmed up to room temperature. These variations are much larger than may be expected from the influence of temperature variations.

A great advantage of the light-chopping method is that it does not detect this varying dark-current, but only its statistical fluctuations, within a narrow frequency band.

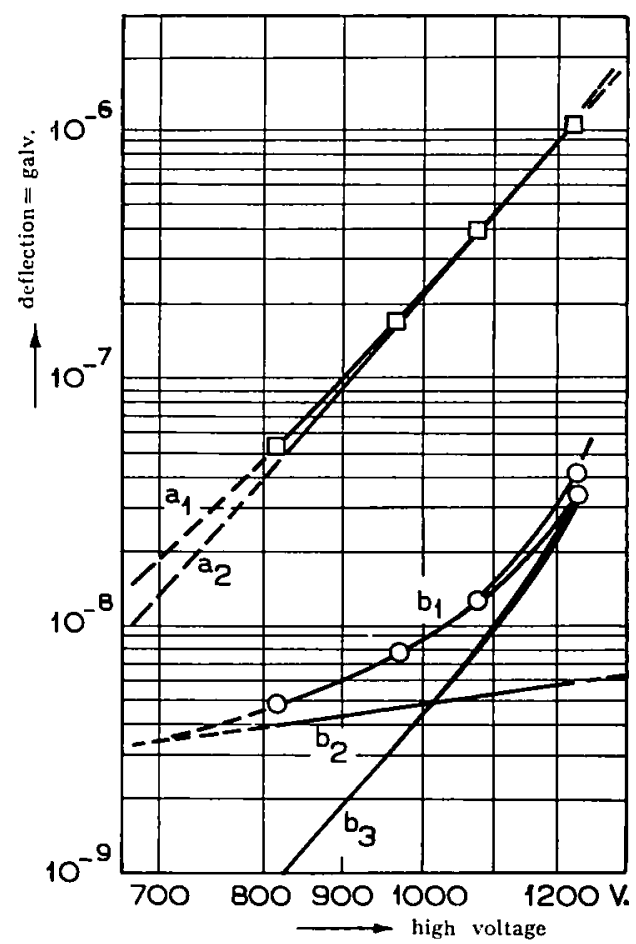

Fig. 3. The d.c. galvanometer deflection as a function of the used voltage over the multiplier for :

$a_{1}$ lightsignal; $a_{2}$ lightsignal after correction for the dark-current; $b_{1}$ dark-current; $b_{2}$ estimated Ohmic leak-current; $b_{3}$ estimated thermionic emission mainly originating from the photocathode $\left(b_{1}=b_{2}+b_{3}\right)$.

In the next table values are given of the dark-current found with a tube with an amplification factor 3 times that of an average tube. For other tubes the same order of magnitude was found for the number of primary darkcurrent electrons. Four independent methods of measuring and different voltages over the stages were used. The values are lower than the ones mentioned by other authors.

The differences are greater than may be explained by the estimated experimental errors, even if slow dark-current variations are taken into account.

The number of dark-current electrons determined with methods, by which 
leak-currents are detected too, have been placed in brackets, because it is incorrect to treat leak currents as photocathode currents. Evidently these numbers are higher, and show that even at high voltages leakcurrents may play an important rôle. Fig. 3 shows very clearly the presence of leak currents at $85 \mathrm{~V}$ en $70 \mathrm{~V}$ per stage. The great importance of using a method which does not detect leak currents, for instance the chopping light method, or a counting device is evident.

With regard to the influence of the voltage drop per stage it may be noted that there is an obvious tendency that the primary dark-current (without leak-current) increases for increasing voltages over the stages. The reasons are field emission and ionization pulses.

VII. Noise of dark-current and photo-current $\left.{ }^{9}\right)^{4}$ ). For the chopping-light method, the following may be remarked with regard to the zero-point noise. The noise of the dark-current limits the useful sensitivity of a multiplier. The r.m.s. of this noise is determined by the formula:

$$
(\Delta I) \equiv \tilde{I}_{0}=\sqrt{2 e \frac{n}{n-1} G I_{0} \Delta t .}
$$

$i=$ charge of an electron

$n=$ mean secondary emission factor

$G=$ amplification factor

$\Delta t=$ effective bandwidth

A narrow bandwidth is desired in measuring weak signals.

In our case $\Delta f \approx 1 \mathrm{~Hz}$. Furthermore it is desirable that the following condition is fulfilled:

$$
20 \frac{n}{n-1} G I_{0} R \gg 1(\text { see } \S 1)
$$

representing the condition that the dark-current noise of the multiplier $\gg$ Johnson noise of the coupling resistor. Experimentally this proved to be true. On account of the relatively low quantum-efficiency $(0.01-0.02$ electron/photon) the statistical fluctuations in the incidence of photons will hardly contribute to the total fluctuations. If the quantum-efficiency is denoted by $q$, the observed noise in the number of photoelectrons leaving the photocathode per sec will approximatively be given by:

$$
\tilde{i_{p h}}=\sqrt{2 e(q+1) i_{p h} \Delta t} \text {, and since } q \sim 0.02 \ll 1,
$$

the fluctuations in the lightflux will not contribute noticeably to the observed noise.

If the noise in the deflection of the a.c. galvanometer, when the multiplier is illuminated with a small, chopped, lightsignal, is caused by the statistical nature of the photo-effect and the thermionic emission process, it would be expected that the square of the total noise consists of two terms, one pro- 
portional to the deflection $u$, the other one is eaqual to the square of the dark-current noise.

Qualitatively this effect is actually found as is shown in fig. 4.

In order to trace this effect quantitatively four records were made with increasing very small light intensities, in such a way that a relatively large noise is present on the recorded deflections. Every record was obtained with a different voltage over the multiplier. After all recorded deflections and noises were reduced to the same amplification factor of the multiplier and the amplifier, it was examined whether:

$$
\frac{r_{u}^{2}-r_{d}^{2}}{u}=\frac{b}{a} \frac{\bar{N}_{p h}}{N_{p h}}=C
$$

where $r_{u}^{2}=b\left(\bar{N}_{p h}+\bar{N}_{d}\right)$ is the square noise in the deflection $u, r_{d}^{2}=b N_{d}$ the zero-point noise and $C$ must be a constant for different lightsignals and voltages over the multiplier. $b$ and $a$ are constants; $b$ contains among other things the bandwidth $B$ of the apparatus.

The r.m.s. noise was estimated with such an accuracy that in the values for $C$ a maximum error of $50 \%$ is possible. Within this accuracy it was found indeed that $C$ is a constant for different signals and voltages. This dark current is calculated from the mean value of $C$, via a fictive deflection caused by it. It shows an evident increase with the voltage over the multiplier. In the next table a summary is given of the numbers found for a certain tube of excessively high $C$. In order to be independent of unexpected effects of

IABLE II

\begin{tabular}{|c|c|c|c|c|c|}
\hline $\begin{array}{l}\text { Voltage per } \\
\text { stage }\end{array}$ & $\begin{array}{c}\text { Reduced } \\
\text { deflection } u\end{array}$ & $\begin{array}{c}\text { Reduced r.m.s. } \\
\text { of the noise }\end{array}$ & $\frac{r_{u}^{2}-r_{d}^{2}}{u}=c$ & $U_{d}=\frac{r_{d}^{2}}{c}$ & $\begin{array}{l}\text { Number of dark } \\
\text { currentelectr. } N_{d}\end{array}$ \\
\hline \multirow[t]{5}{*}{$70 \mathrm{~V}$} & 0 & 0.30 & & \multirow[t]{5}{*}{0.75} & \multirow[t]{5}{*}{750 per sec. } \\
\hline & 0.36 & 0.36 & 0.108 & & \\
\hline & 1.1 & $0.39^{5}$ & 0.066 & & \\
\hline & 2.0 & 0.55 & 0.105 & & \\
\hline & 3.7 & 0.59 & 0.070 & & \\
\hline \multirow[t]{5}{*}{$85 \mathrm{~V}$} & 0 & $0.42^{5}$ & & \multirow[t]{5}{*}{1.5} & \multirow[t]{5}{*}{1500} \\
\hline & 0.37 & 0.47 & 0.055 & & \\
\hline & 1.2 & 0.49 & 0.050 & & \\
\hline & 1.9 & 0.70 & 0.140 & & \\
\hline & 3.7 & 0.85 & 0.140 & & \\
\hline \multirow{5}{*}{$100 \mathrm{~V}$} & 0 & 0.49 & & \multirow{5}{*}{2.0} & \multirow{5}{*}{2000} \\
\hline & 0.3 & 0.51 & 0.072 & & \\
\hline & $1.0^{5}$ & 0.62 & 0.140 & & \\
\hline & 1.9 & 0.78 & 0.190 & & \\
\hline & $3.6^{5}$ & 0.78 & 0.100 & & \\
\hline \multirow{6}{*}{$115 \mathrm{~V}$} & 0 & 0.53 & & \multirow[t]{6}{*}{$2.3^{3}$} & \multirow[t]{6}{*}{2350} \\
\hline & 0.37 & 0.55 & 0.070 & & \\
\hline & 0.95 & 0.62 & 0.105 & & \\
\hline & 1.95 & 0.91 & 0.270 & & \\
\hline & 3.7 & 0.91 & 0.140 & & \\
\hline & & & $\bar{c} \approx 0.12$ & & \\
\hline
\end{tabular}


the apparatus the square of the recorded noise was put proportional to the number of primary electrons with another proportionallity factor as the deflections.

$U_{d}$ is the fictive reduced deflection caused by the dark-current if it had been modulated and recorded in the same way as the $50-\mathrm{Hz}$ modulated photocurrent. From the known sensitivity of the instruments it is calculated that 1 $\mathrm{cm}$ reduced deflection corresponds with an unmodulated primary current of 1000 electr./sec.

So in this way an exceptionally low dark current of the magnitude of 1000 primary electr./sec was found. Furthermore it is easily seen that the noise found in this way is indeed proportional to, but (after taken into account statistical fluctuations in the secondary emission factor) not equal to the root of the number of primary electrons, the factor of proportion for most tubes being $>2-3$.

Possible reasons for this behaviour are:

1. Losses by incomplete focussing; if for instance only one half of the number of electrons falls on the first dynode, the noise relatively increases a factor $\sqrt{ } 2$. (By the use of the multiplier as a phototube ( $\S 3$. III) there were no such losses).

2. Patch structures of the secondary emission surfaces. Estimation of the influence of such a structure of the first dynode on the noise gives a possible enhancement of the noise of a factor 2 .

3. Flicker effect. In the case that the enhancement of the noise is caused by the Flicker effect, no such effects must be found for frequencies $\gg 50 \mathrm{~Hz}$. Research in this direction is being made *).

The circumstance that the dark-current is about ten times smaller than found with another method and from the litterature, whereas the noise is 2-3 times larger than corresponds with the number of dark-current electrons per sec raises the surmise that the dark current found in this way is to low, but not so the noise. In that case all differences should be explained as inherent to the method of measuring. However, for various tubes, different factors are found $(2-7)$. This gives an indication that most probably the apparatus does not cause this effect.

Moreover, the noise in the photo-current is also found to be 2-3 times larger than is expected from the mentioned formulas. A glance at fig. 4 shows that no errors were made in the calculation.

When the dark current pulses are counted, a fast amplifier must be used to count the pulses separately. The fluctuation phenomena here are somewhat different, i.e. the fluctuations in the number of counted pulses during the time of the measurement. The sensitivity here is limited by the fluctuations in the number of counted pulses of the dark current. As the time of measure-

\footnotetext{
*) Measurements of R. Rits m a, in this laboratory, of the noise of vacuum photo-tubes at $\mathrm{O}-\mathrm{Hz}$ give an enhancement of the noise of a factor 10 , whereas the integrated noise over all frequences show no discrepancy, so that a Flicker effect seems to be probable.
} 


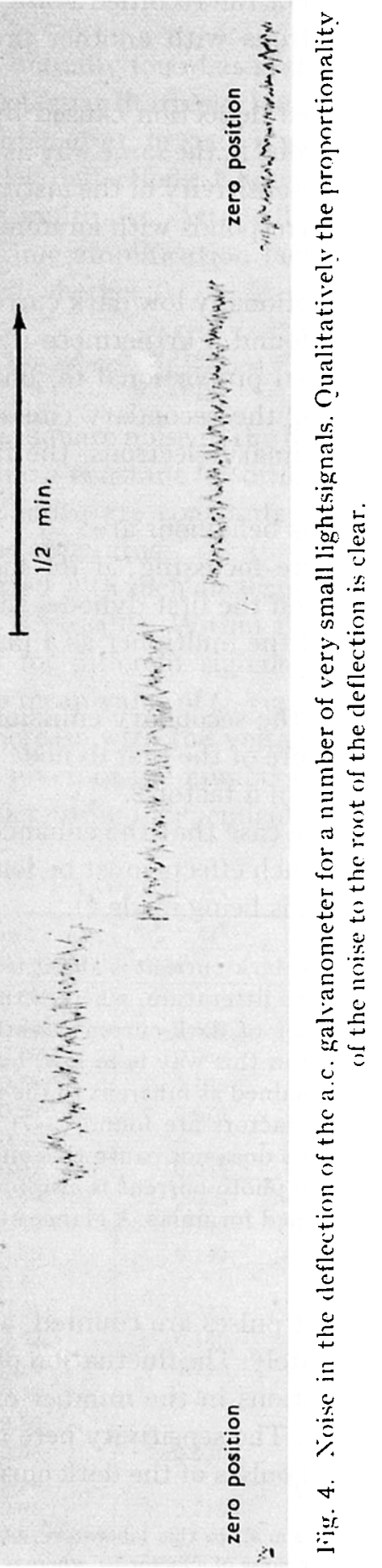


ment is usually rather long $(30-120 \mathrm{sec})$ these fluctuations should be low when they were purely statistical. However the dark-current has a very

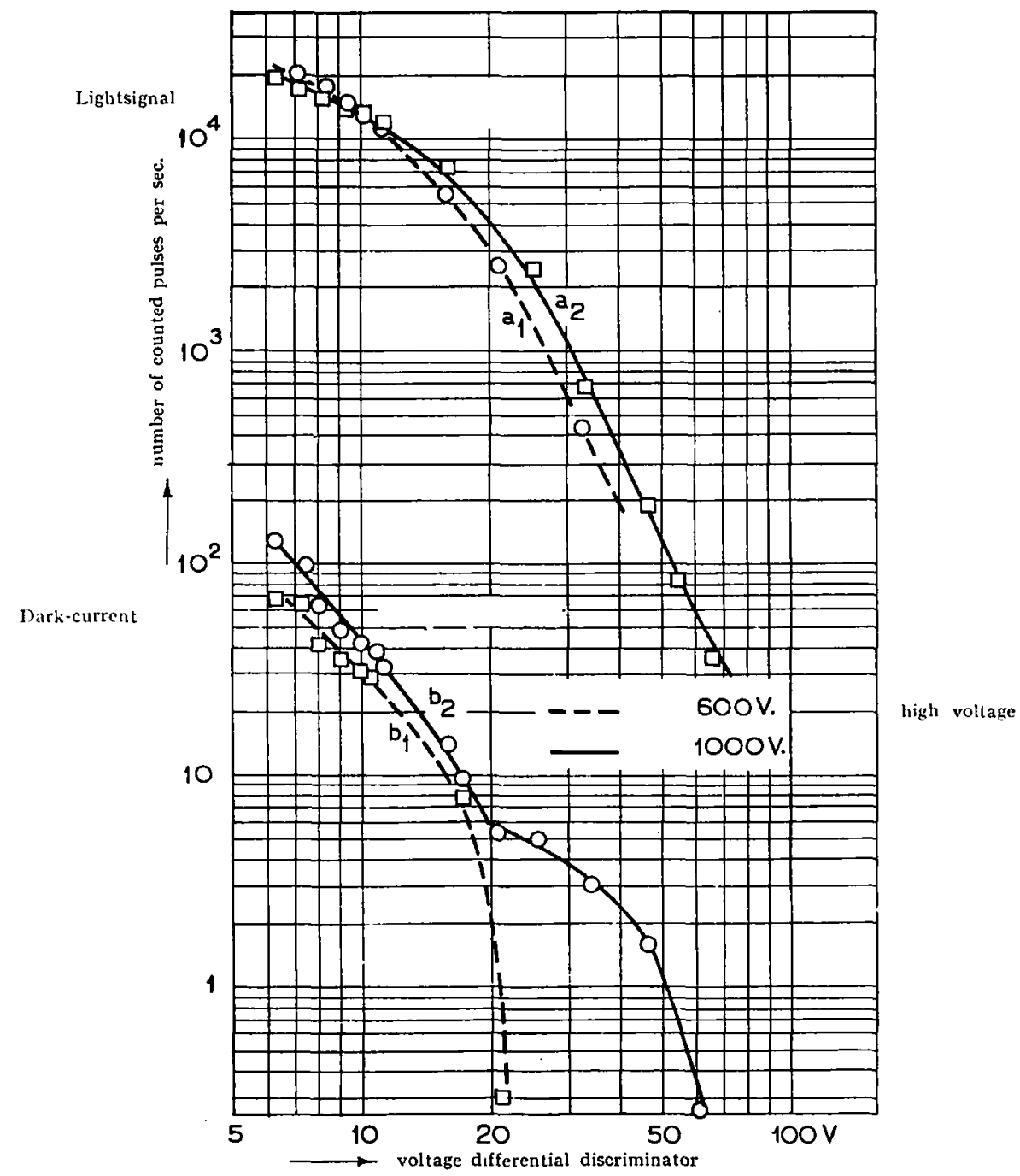

Fig. 5. Pulse-height distribution.

" for a lightsignal when a low voltage is used.

$a_{2}$ the same with a high voltage, $b_{1}$ and $b_{2}$ the same for the dark-current.

The increase in internal amplification has been compensated by decrease in sensitivity of the broadband-amplifier. The decrease in the signal-to-noise ratio with the higher voltage and the occurrence of ion-pulses is clearly seen.

irregular slow drift, so that correction for the dark-current by substracting leaves rather a large error of for instance $10 \%$ of the dark current. In this case the light chopping method is the more sensitive one. It is possible, 
however, to combine the counting method with the light-chopping method by using a two channel counting device, so that one register counts only the illuminated periods, the second one only the dark periods ${ }^{17}$ ). In this case both methods will be equivalent, the longer counting time being canceled by an equal long time of recording of the galvanometer deflection.

Without any correction for the dark-current it is often possible to reduce its disturbing influence by high discriminating, provided that the voltage per stage is less than $80 \mathrm{~V}$.

Fig. 5 shows that the pulse-distribution curves of photo- and dark-current are somewhat different, because the photo-current is generated exclusively at the photocathode, the dark-current is also generated at the following dynodes. Therefore in the photocurrent relatively more high pulses are present, so that high discrimination is advantageous.

VIII. Optimum voltages over the stages. The field on the cathode effects the magnitude of the primary dark-current from the photocathode and first dynodes too. First the workfunction is lowered by the field, but probably of more importance is the fact that the photocathode has essentially a patch structure, so that field-emission processes are possible too.

If the voltage over the multiplier is enhanced the output dark-current increases more than agrees with the increase in the amplification factor.

The signal to noise ratio decreases therefore if the voltage over the stages is enhanced; a voltage of $60 \mathrm{~V}-70 \mathrm{~V}$ proved to give the best signal to noise ratio, as it is necessary to take care that the multiplier noise remains $\gg$ amplifier noise.

With the counting device the following result was obtained $\left.{ }^{4}\right)$. In the range of $60 \mathrm{~V}-80 \mathrm{~V}$ per stage a pulse distribution curve was found for the darkcurrent nearly similar in shape to that of photopulses. Beginning at $80 \mathrm{~V}$ per stage and very evidently at $100 \mathrm{~V}$ per stage an alteration in the shape of the dark-current pulse distribution curve was found (not so in the photopulse distribution curve). This curve is built up of two curves: a normal one, as found for photopulses and for dark-current pulses at low voltages and a curve of relatively few but very high pulses (fig. 5).

Most probably this is a beginning of the ionisation phenomena, which increase very rapidly with the voltage and cause the ion feed back that make most multipliers unstable at voltages higher than $110 \mathrm{~V}$ per stage.

The appearance of these wings on the distribution curve makes it impossible to discriminate anyhow against the dark-current, but may make it advantageous to use a differential-discriminator, when it is necessary to use high voltages. In order to get a high signal to noise ratio and still a great amplification factor it was tried to diminish only the voltage over the first stage(s) because the dark-current is mainly generated here. In doing this the signal to noise ratio decreased however, probably because focussing losses eliminated the experted advantage. Enlarging of the voltage over the first stage(s) 
caused likewise a decreasing signal to noise ratio, so that a nearly uniform division of the voltage over the stages gives probably the best signal to noise ratio.

IX. Cooling with liquid a i $\left.\mathrm{r}^{4}\right)^{13}$ ). Because for our measuring apparatus at low voltages the dark-current is principally determined by the thermionic emission from the photocathode and first dynodes, it is suitable to use the multiplier at reduced temperatures; the thermionic emission $I_{t}$ will then reduce according to the formula of Richardson: $I_{t} \sim T^{2} \exp -w / k T$ ( $T=$ abs. temp., $w=$ work function; $k=$ Boltzmann constant). The cooling was obtained in the following arrangement:

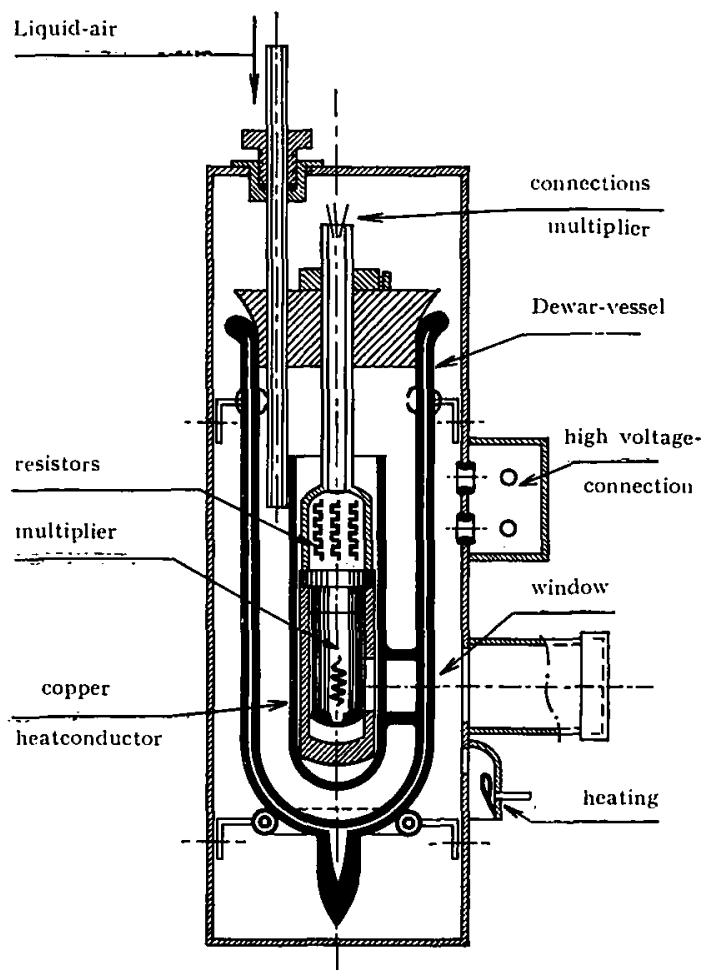

Fig. 6. Vessel for cooling multiplier.

In a Dewar vessel (fig. 6) an opening of $3 \mathrm{~cm}$ diameter is provided inside. A glass tube is melted on this hole and opposite the window so formed the silver coating is rubbed from the exterior tube of the Dewar vessel, to make it possible for the light to fall on the cathode of the multiplier. The resistors are fitted up in a crown on the sockel of the multiplier and are enclosed by a copper hat suspended from a perspex bar. In this bar a narrow tube is provided for the electrical connections (earth, output, voltage) to the multiplier which are realised by very thin wires to make the heat flow to the multiplier 
as small as possible. The multiplier is painted black, but heat transfer by radiation was not sufficient to reach $-190^{\circ} \mathrm{C}$ (about $-100^{\circ} \mathrm{C}$ was reached). Therefore the multiplier is enclosed by a thick copper tube, so that heat conduction at lower temperatures takes over the effects of radiation. The heat production in the resistors is about $0.4 \mathrm{Watt}$, but this gives no difficulties; $500 \mathrm{~mL}$ is sufficient for 10 hours cooling; the operation is very stable; liquid air is easily filled by.

For the alternating light method the noise proved to be reduced by a factor 20-25, which would mean a reduction of the dark-current of 500 times (fig. 7). Only a few electrons a second remain.

noise at room temperature

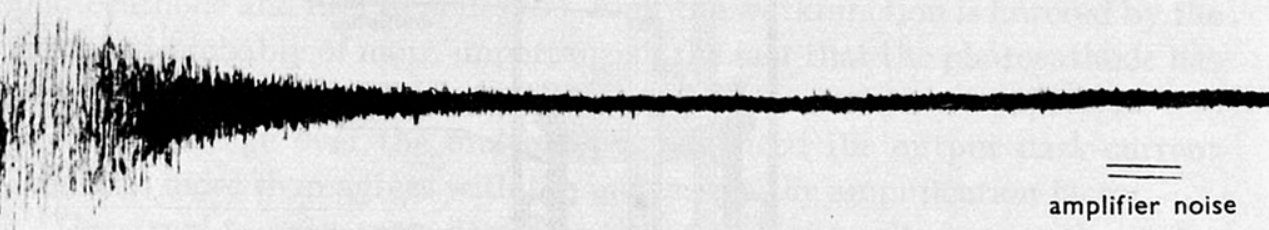

$\downarrow$

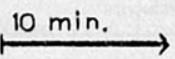

lïr. 7. Mark-current noise diminishing when the multiplier is cooled to licpuicl-air temperature.

However, this factor is less than one should expect from the Richardson formula. From this formula a work function of $0.2 \mathrm{el}^{\prime}$ should be expected, whereas from the threshold wavelength a workfunction of $1.5 \mathrm{eV}$ is found. Most probably this may be explained by assuming that on the photocathode little regions are present with a smaller workfunction than the rest of the photocathode, which regions are responsible principally for the thermionic emission at reduced temperature. At $300^{\circ} \mathrm{K}$ the thermionic emission will be determined by the whole cathode, which may explain the irregular behaviour of the dark-current. Other indications for a patch structure were already the abnormal behaviour of the noise and the variable sensitivity over the photocathode.

For the alternating light device cooling seems not so very suitable, because the small dark-current present at room temperature amounts to about 1000 electrons/sec which means an error in the measurements of a photo-current of 1000 electrons $\mathrm{sec}$ of $4-5 \%$ (apart from the fact that the noise proved to be 
2-3 times too large). Without dark-current (after cooling) an error of $3 \%$ should be present.

For a counting device without dark-current correction on the contrary cooling is very useful; most of the thermionic electrons come from the photocathode and first stages, and are counted. If the number of dark-current pulses is reduced 500 times, the lowest detectable light level becomes 500 times smaller. By counting during long times (e.g. 5 minutes) lightsignals of $10^{-16}$ watt may be detected with an accuracy of $50 \%$ (corresponding to photo-currents of 1-2 electrons/sec from the cathode).

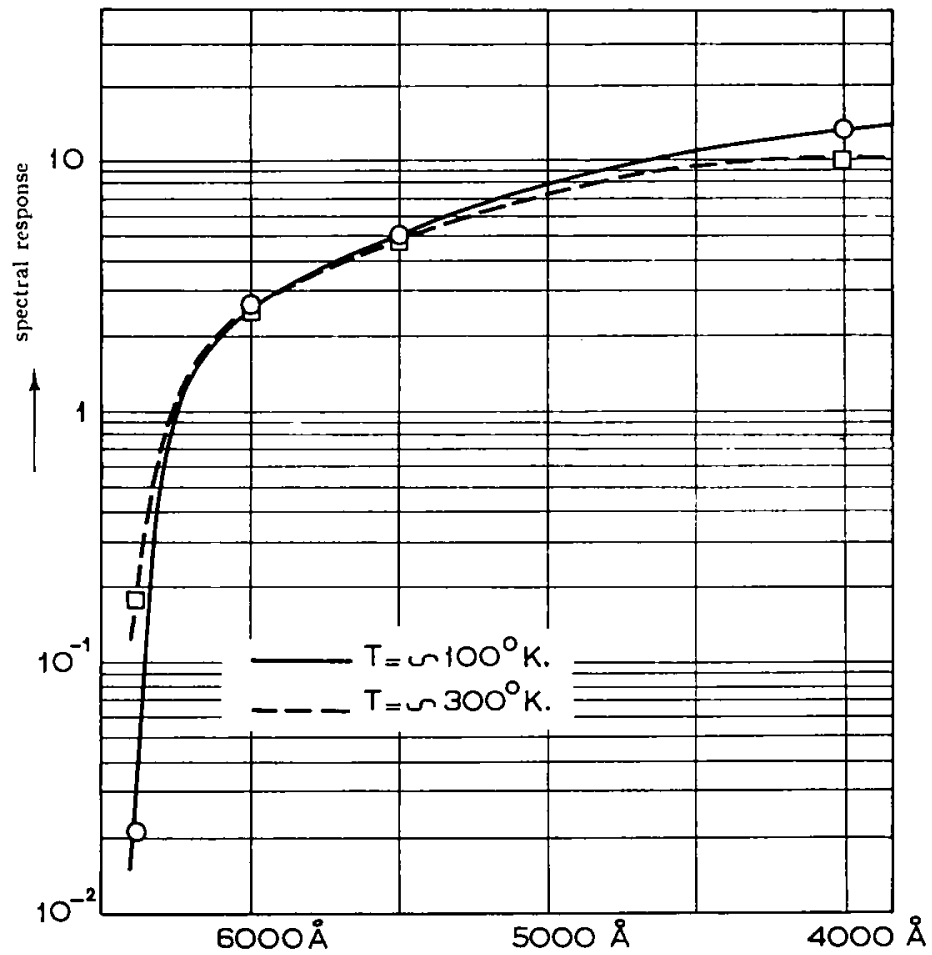

Fisr. 8. Alteration of the spectral response curve when the multiplier is corlect to liquid-air temperature.

At reduced temperatures a reduction of the sensitivity for "white" light of a factor 2 was found. Wavelength sensitivity curves were determined before and after the cooling (fig. 8) ${ }^{14}$ ). It turned out that the sensitivity for red light $(6500 \AA)$ was decreased by a factor 8 , whereas the sensitivity for blue light $(4000 \AA-4500 \AA)$ increased $40 \%$.

Theoretically this can be explained as follows: The sensitivity curve at $6500 \AA$ (threshold wavelength) is determined principally by the surface effect, the sensitivity at $4000 \AA$ (optimum range) by the volume effect. The decreased sensitivity for long wavelengths may be interpreted as a regression 
of the Fermi tail. The increase in the sensitivity for short wavelength is caused by the enlargement of the mean free path of the metal electrons at lower temperatures. Wit the volume effect the yield is determined by the light absorption in a layer of the length of the mean free path. At reduced temperature the free path increases, whereas the absorption remains unchanged. So the yield in this region of the sensitivity curve will increase at reduced temperature.

Acknowledgement. The authors wish to express their appreciation to $\mathrm{Mr}$. C. Th. J. A l k e m a d e for his valuable advice and criticism in the preparation of this paper.

Received 30-1-54.

\section{REFERENCES}

1) Milat 7., J. M. W. and Ornste in, L. S., Physica 2 (1935) 355.

.) M i l a $t z$, J. M. W., Thesis Utrecht 1937.

3) M i l a t z, J. M. W. and B loem berge n, N., Physica 11 (1946) 449.

4) B o e schote n, F., Thesis Utrecht 1953.

5) Bonfiglioli, G. and Mom t a len ti, G., Spec. Acta 5 (1953) 371 .

6) Engstrom, R. W., J. opt. Soc. Am. 37 (1947) 420.

7) M orton, G. A., R C A Rev. 10 (1949) 525.

8) Schaet $\mathrm{Si}, \mathrm{N}$. and $\mathrm{B}$ a u $\mathrm{mg}$ a $\mathrm{r} \mathrm{t}$ er, W., Helv. phys. Acta 25 (1952) 605.

9) Shockley, N. and Pierce, J. R., Proc. I.R.E. 26 (1936) 231.

10) liessler, K. G. and Wolfe, R. A., J.opt. Soc. Am. 37 (1947) 133.

11) H a sler, M. F., I, in d hurst, R. W. and K e m p, J. W., J. opt. Soc. Am. :38 (1948) 789.

12) Di eke, G. H., A rapport for the Office of Prod. and Res. of the War Prod. Board: Photoelectric Intensity Measurements in spectra. John Hopkins University.

13) S a u n erson, J. L., Calde court, V. J. and Peterson, E. W., J. opt. Soc. Am. 35) (1945) 681 .

14) Gilmore, E. H. and Ku ipe, R. H., J. opt. Soc. Am. 12 (1952) 481.

15) M a c q, M., Congrès du G.A.M.S. Paris, 20-22 Juin (1951).

16) M i 1 a $\mathrm{t} z$, J. M. W., Ludt, P. M., Alkem ade, C. T. J., and Olink, J. I., Physica $14(1948) 260$.

17) Smit, J. A., Milat \%. J. M. W. and S m i t, C., Physica 20 (1954) 39. 